\title{
Supramolecular Aggregations through the Inclusion Complexation of Cyclodextrins and Polymers with Bulky End Groups
}

\author{
SHIH-CHI CHAN, ${ }^{1}$ SHIAO-WEI KUO, ${ }^{1}$ HWO-SHUENN SHE, ${ }^{2}$ HO-MAY LIN, ${ }^{1}$ HSIN-FANG LEE, ${ }^{1}$ FENG-CHIH CHANG ${ }^{1}$ \\ ${ }^{1}$ Institute of Applied Chemistry, National Chiao Tung University, Hsin Chu, Taiwan \\ ${ }^{2}$ National Synchrotron Radiation Research Center, Hsinchu Science Park, Taiwan
}

Received 19 May 2006; accepted 28 September 2006

DOI: 10.1002 / pola.21820

Published online in Wiley InterScience (www.interscience.wiley.com).

\begin{abstract}
Mono-polyhedral oligomeric sillsesquioxane-end capped poly( $\varepsilon$-caprolactone) (mPPCL) can form inclusion complexes (ICs) with $\alpha$ - and $\gamma$-cyclodextrins (CDs) but not with $\beta$-CD. These CD ICs have been characterized with X-ray diffraction, solid-state ${ }^{13} \mathrm{C}$ cross-polarization/magic-angle-spinning NMR spectroscopy, ${ }^{1} \mathrm{H}$ NMR spectroscopy, Fourier transform infrared spectroscopy, differential scanning calorimetry, thermogravimetric analysis, and scanning electron microscopy. The poly(e-caprolactone) (PCL) chain of $\mathrm{mPPCL}$ is included within the channel provided by the CDs to form a columnar, crystalline structure. The PCL/CD ratios determined by ${ }^{1} \mathrm{H}$ NMR spectroscopy for the ICs with $\alpha$ - or $\gamma$-CDs are higher than the stoichiometries because of the steric hindrance of the bulky polyhedral oligomeric silsesquioxane chain end and result in a fraction of the $\varepsilon$-caprolactone units free from complexation with the CDs. On the basis of these analyses, we propose some possible structures for these CD/mPPCL ICs. ( $) 2006$ Wiley Periodicals, Inc. J Polym Sci Part A: Polym Chem 45: 125-135, 2007

Keywords: inclusion complexes; nanocomposites; polyhedral oligomeric silsesquioxane (POSS); polyrotaxanes; supramolecular structures
\end{abstract}

\section{INTRODUCTION}

The progress in supramolecular chemistry has introduced a fascinating new field of supramolecular architecture, and a number of nanostructured supramolecular assemblies have been discovered. Inclusion complexes (ICs) consisting of cyclic molecules and polymeric chains have been recently prepared as novel molecular assemblies. $^{1-5}$ Cyclodextrins (CDs) have been widely used as host molecules for polyrotaxanes and

Correspondence to: S.-W. Kuo (E-mail: kuosw@mail.nctu. edu.tw)

Journal of Polymer Science: Part A: Polymer Chemistry, Vol. 45, 125-135 (2007) (ㅇ) 2006 Wiley Periodicals, Inc. pseudopolyrotaxanes because they can include various chain polymers. CDs are cyclic oligosaccharides built from 1,4-glucopyranose units that consist of six $(\alpha-)$, seven $(\beta-)$, or eight $(\gamma-)$ glucose units processing a torus-shaped structure with a hydrophilic exterior and a hydrophobic cavity. ${ }^{6}$ They have rigid, well-defined structures and can act as hosts to form a large variety of supramolecules. ${ }^{7}$ Supramolecular ICs stabilized by noncovalent interactions can be formed by the threading of CD molecules onto various polymer chains. The ability to insert polymer chains into the narrow, cylindrical, crystalline channels of CDs is well known. ${ }^{8-13}$ The inclusion of a polymer chain within the CD cavity is entropically unfavorable, and this is promoted by intermole- 
cular hydrogen bonding between neighboring $\mathrm{CDs}$ and noncovalent attractive interactions between the chain and CD, such as van der Walls and hydrophobic interactions. ${ }^{14-16}$

Harada and Kamachi ${ }^{17}$ prepared the first IC in 1990; many cyclic molecules were threaded onto a linear polymeric chain capped with bulky blocking groups, which consisted of $\mathrm{CD}$ and poly(ethylene oxide) (PEO). Since this initial discovery of $\mathrm{CD} /$ polymer ICs, several reports have described how many linear polymeric guests, possessing either hydrophilic or hydrophobic properties, are able to form ICs with different types of CDs. ${ }^{8-13}$ To form an IC, the size correlation between the cross-sectional area of the polymer chain and the cavity internal diameter of CDs is a key factor. ${ }^{18}$ The geometries of CDs have a depth of approximately $7.5 \AA$ and internal diameters of about $4.5,7.0$, and $8.5 \AA$ for $\alpha$-, $\beta$-, and $\gamma$-CDs, respectively. ${ }^{16}$ They can form ICs only with guest molecules of the proper sizes. Because of the long-chain nature of the polymer, the crystal structure of a polymer/CD complex is expected to be columnar. ${ }^{19}$ Their cylindrical structures, with cavities about $0.75 \mathrm{~nm}$ deep and a $0.5-0.8-\mathrm{nm}$ inside diameter, yield various unique properties. Such a structure confines the conformation of the polymer chain to a rodlike one (all-trans configuration) because of the very small cavities and close packing of the CD molecules. As Tonelli and coworkers ${ }^{20,21}$ indicated, the study of these types of ICs provides an opportunity to investigate the behavior of single polymer chains or two adjacent parallel chains in isolated and well-defined environments.

A polyhedral oligomeric silsesquioxane (POSS) reagent is unique in both its chemical composition and physical nature. ${ }^{22-25}$ The rigid and thermally stable silicon-oxygen skeleton core is able to bind more than one hydrocarbon outer arm. ${ }^{26}$ POSS derivatives are a family of molecularly precise, nearly isotropic molecules that have diameters ranging from 1 to $3 \mathrm{~nm}$, depending on the number of silicon atoms in the central cage and the nature of the peripheral substituent groups. ${ }^{27} \mathrm{~A}$ cubic silsesquioxane unit possesses inorganic $\mathrm{Si}_{8} \mathrm{O}_{12}$ core surrounded by eight tunable substituent groups $\left(\mathrm{T}_{8}\right)$. Because of their unique structures, POSS derivatives are useful building blocks for the preparation of nanostructured materials. We are interested in combining the well-defined macromolecular architectures of POSS and CDs to generate polymeric building blocks with distinct three-dimensional shapes for the self-assembly of supramolecular structures. On the other hands, biodegradable polyesters have been intensively investigated because of their potential importance in a number of different applications. ${ }^{28}$ Poly( $\varepsilon$-caprolactone) (PCL) is one of the most attractive and promising synthetic polyesters. PCL is able to form columnarstructured ICs with both $\alpha$-CD and $\gamma$-CD in high yields. In the $\gamma$-CD/PCL complex, two adjacent PCL chains are threaded within each $\gamma$-CD channel. ${ }^{29}$

Recently, supramolecular chemistry based on POSS has attracted much attention because of not only the obvious elegance of the systems but also the applications for photoresists, ${ }^{30}$ biomacromolecules, ${ }^{31}$ and liquid crystals. ${ }^{32}$ For the formation of a CD/POSS supramolecular complex, we have reported the formation of starlike ICs between POSS-cored star PCL and CDs. ${ }^{33}$ In this study, we synthesized mono-polyhedral oligomeric sillsesquioxane-end capped PCL (mPPCL) to investigate the effect of the POSS moiety on the resultant ICs between $\mathrm{MPPCL}$ and CDs. Here we have found that MPPCL can form ICs to give polypseudorotaxanes with $\alpha$ - and $\gamma$-CDs despite the bulky POSS moiety capped on one end of the linear PCL. In this article, we report the preparation and characterization of ICs of CDs with synthesized mPPCL. To the best of our knowledge, the morphologies of the supramolecular self-assembly structures of these $\alpha$ $\mathrm{CD} / \mathrm{mPPCL}$ and $\gamma$-CD/mPPCL ICs have never been reported previously.

\section{EXPERIMENTAL}

\section{Materials}

Trisilanolisobutyl-POSS was purchased from Hybrid Plastics. 2-(Trichlorosilyl)ethyl acetate, triethylamine, and tin(II) 2-ethylhexanoate were obtained from Aldrich. $\varepsilon$-Caprolactone ( $\varepsilon$-CL) was obtained form Acros Organics. $\alpha$-CD and $\gamma$-CD were obtained from Tokyo Kasei (Tokyo, Japan). All CDs were dried at $80^{\circ} \mathrm{C}$ in vacuo for 2 days before use. Other chemicals were used as received without further purification.

\section{Synthesis of Isobutyl-POSS Ethyl Acetate}

The POSS triols $\left[\left(i-\mathrm{C}_{4} \mathrm{H}_{9}\right)_{7} \mathrm{Si}_{7} \mathrm{O}_{9}(\mathrm{OH})_{3} ; 8.7 \mathrm{~g}\right.$, $12.9 \mathrm{mmol}]$ and triethylamine $(3.38 \mathrm{~g}, 41.25 \mathrm{mmol})$ were dissolved in $125 \mathrm{~mL}$ of tetrahydrofuran 


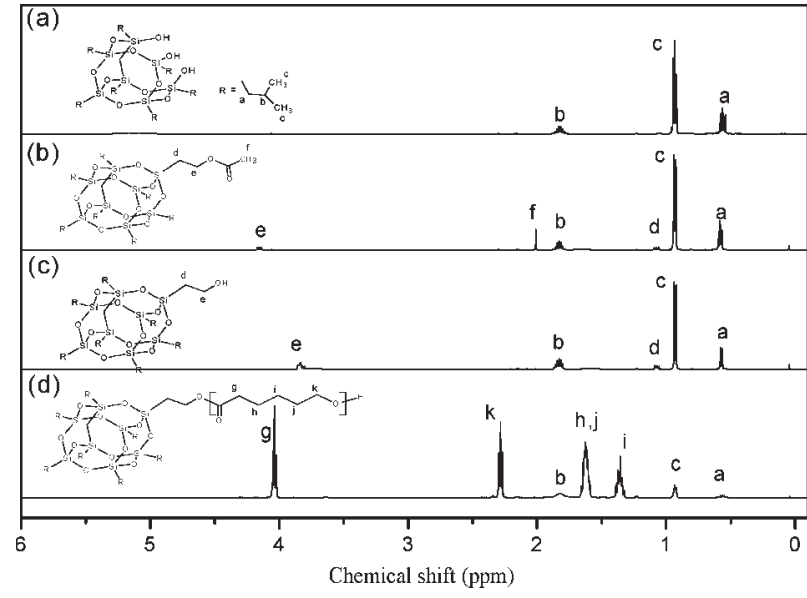

Figure 1. ${ }^{1} \mathrm{H}$ NMR spectra of (a) trisilanolisobutylPOSS, (b) isobutyl-POSS ethyl acetate, (c) hydroxyethylisobutyl-POSS, and (d) mPPCL.

(THF). The solution was cooled in an ice bath, and a THF solution of 2-(trichlorosilyl)ethyl acetate $(2.25 \mathrm{~mL}, 12.9 \mathrm{mmol}, 0.516 \mathrm{M})$ was added dropwise over a 25 -min period to the stirring solution. The mixture was allowed to warm to room temperature and reacted for $6 \mathrm{~h}$. The resultant mixture was filtered to remove $\mathrm{Et}_{3} \mathrm{~N}-$ $\mathrm{HCl}$, and the filtrate was then precipitated into methanol directly. After filtration and drying in vacuo, a white powder was afforded (87\%). Selected characterization data are as follows.

${ }^{1} \mathrm{H} \mathrm{NMR}\left(500 \mathrm{MHz}, \mathrm{CDCl}_{3}, \mathrm{CHCl}_{3}\right.$ as the reference): $4.15\left(\mathrm{t}, 2 \mathrm{H},-\mathrm{CH}_{2}-\mathrm{O}\right), 2.01(\mathrm{~s}, 3 \mathrm{H}$, $\left.-\mathrm{CO}-\mathrm{CH}_{3}\right), 1.84\left[\mathrm{~m}, 1 \mathrm{H},-\mathrm{CH}-\left(\mathrm{CH}_{3}\right)_{2}\right], 1.07$ (t, $\left.2 \mathrm{H},-\mathrm{CH}_{2}-\mathrm{CH}_{2}-\mathrm{O}\right), 0.95[\mathrm{~m}, 6 \mathrm{H},-\mathrm{CH}-$ $\left.\left(\mathrm{CH}_{3}\right)_{2}\right], 0.58\left[\mathrm{~m}, 2 \mathrm{H}, \mathrm{CH}_{2}-\mathrm{CH}-\left(\mathrm{CH}_{3}\right)_{2}\right]$. Fourier transform infrared (FTIR; $\mathrm{KBr}, \mathrm{cm}^{-1}$ ) $v$ $\mathrm{C}-\mathrm{H}: 2959 \mathrm{~s}, 2931 \mathrm{~s}, 2875 \mathrm{~m} ; v \mathrm{C}=\mathrm{O}: 1725 \mathrm{~s} ; \delta$ $\mathrm{CH}_{3}: 1258 \mathrm{~m} ; v \mathrm{Si}-\mathrm{C}: 1173 \mathrm{~m} ; v \mathrm{Si}-\mathrm{O}: 1082 \mathrm{~s}$; $v \mathrm{C}-\mathrm{O}: 1010 \mathrm{~s}$ \{displayed in Figs. 1(b) and 2(b); the ${ }^{1} \mathrm{H}$ NMR and FTIR spectra of the pure POSS triols $\left[\left(i-\mathrm{C}_{4} \mathrm{H}_{9}\right)_{7} \mathrm{Si}_{7} \mathrm{O}_{9}(\mathrm{OH})_{3}\right]$ are presented in Figs. 1(a) and 2(a)\}.

\section{Synthesis of Hydroxyethylisobutyl-POSS by Base Hydrolysis}

Isobutyl-POSS ethyl acetate (3 g) was dissolved in a mixture of 1,4-dioaxane and $30 \%$ aqueous $\mathrm{NaOH}(10: 1)$. The mixture was stirred overnight at the ambient temperature. The solution was then precipitated into excess methanol to afford a white solid that was subsequently dissolved in $10 \mathrm{~mL}$ of ether and extracted with water twice.
After dehydration and drying in vacuo, the yield of the white powder was $71 \%$. Selected characterization data are as follows.

${ }^{1} \mathrm{H}$ NMR $\left(500 \mathrm{MHz}, \mathrm{CDCl}_{3}, \mathrm{CHCl}_{3}\right.$ as the reference): $3.58\left(\mathrm{t}, 2 \mathrm{H},-\mathrm{CH}_{2}-\mathrm{OH}\right), 1.84[\mathrm{~m}, 1 \mathrm{H}$, $\left.-\mathrm{CH}-\left(\mathrm{CH}_{3}\right)_{2}\right], 1.07\left(\mathrm{t}, 2 \mathrm{H},-\mathrm{CH}_{2}-\mathrm{CH}_{2}-\mathrm{O}\right)$, $0.95\left[\mathrm{~m}, 6 \mathrm{H},-\mathrm{CH}-\left(\mathrm{CH}_{3}\right)_{2}\right], 0.58\left[\mathrm{~m}, 2 \mathrm{H}, \mathrm{CH}_{2}-\right.$ $\left.\mathrm{CH}-\left(\mathrm{CH}_{3}\right)_{2}\right]$. FTIR $\left(\mathrm{KBr}, \mathrm{cm}^{-1}\right)$ v $\mathrm{OH}: 3318 \mathrm{~s}$; $v \mathrm{C}-\mathrm{H}: 2959 \mathrm{~s}, 2931 \mathrm{~s}, 2875 \mathrm{~m} ; \delta \mathrm{CH}_{3}: 1258 \mathrm{~m}$; $v \mathrm{Si}-\mathrm{C}: 1173 \mathrm{~m} ; v \mathrm{Si}-\mathrm{O}: 1082 \mathrm{~s} ; v \mathrm{C}-\mathrm{O}: 1010 \mathrm{~s}$ [presented in Figs. 1(c) and 2(c)].

\section{Synthesis of $\mathrm{mPPCL}$}

The initiator hydroxyethylisobutyl-POSS $(0.437 \mathrm{~g}$, $0.5 \mathrm{mmol}$ ) was placed in a Schlenk flask equipped with a reflux condenser and a magnetic stirrer. $\varepsilon$-CL $(2.5 \mathrm{~mL}, 25 \mathrm{mmol})$ was added to dissolve the initiator with a monomer/initiator ratio of 50/1. The mixture was heated to $115{ }^{\circ} \mathrm{C}$, and $\mathrm{Sn}(\mathrm{Oct})_{2}(0.1 \mathrm{mmol})$ was added. The bulk reaction began to reflux immediately under nitrogen for $24 \mathrm{~h}$. The mixture was then diluted with excess THF and precipitated into cold methanol to give a high yield of a white, crystalline powder [number-average molecular weight $\left(M_{\mathrm{n}}\right)$ $=6100 \mathrm{~g} / \mathrm{mol}$, polydispersity index $(\mathrm{PDI})=1.2]$. The ${ }^{1} \mathrm{H}$ NMR and FTIR spectra of the synthesized organic/inorganic mPPCL are presented in Figures 1(d) and 2(d), respectively.

\section{Preparation of the ICs}

mPPCL (0.2 g; $1.48 \mathrm{mmol}$ of the monomer unit) was first dissolved in acetone $(10 \mathrm{~mL})$ by sonifi-

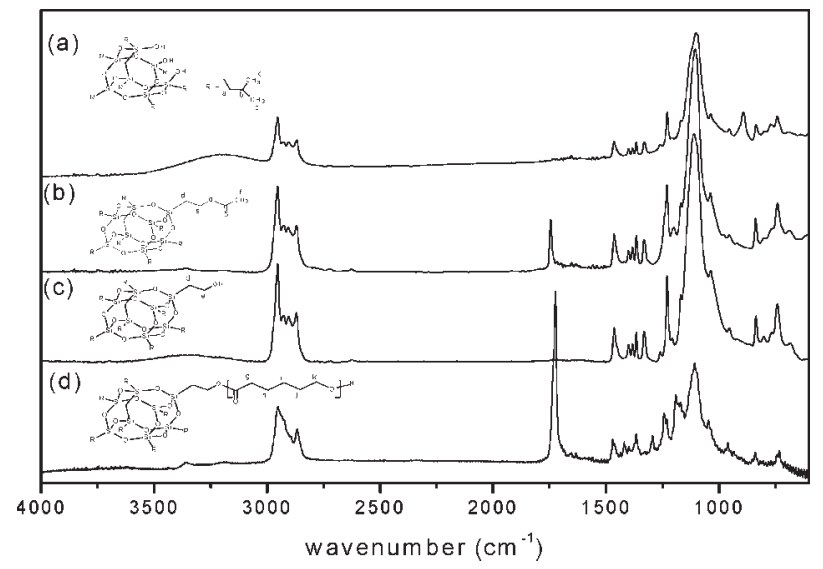

Figure 2. FTIR spectra of (a) trisilanolisobutylPOSS, (b) isobutyl-POSS ethyl acetate, (c) hydroxyethylisobutyl-POSS, and (d) mPPCL. 
cation. A saturated aqueous solution of $\alpha-\mathrm{CD}$ (12 $\mathrm{mL}, 1.8 \mathrm{mmol}$ ) was added, and the heterogeneous mixtures were ultrasonically agitated for $30 \mathrm{~min}$ with heating and then left to stand overnight at room temperature. The white powder was collected by centrifugation and then washed with water and acetone to remove any free polymer and uncomplexed CD. Finally, the IC was dried in a vacuum oven at $40{ }^{\circ} \mathrm{C}$ for $48 \mathrm{~h}$. For $\gamma$-CD, mPPCL $(0.2 \mathrm{~g} ; 1.48 \mathrm{mmol}$ of the monomer unit) and a saturated aqueous solution of $\gamma$-CD $(10 \mathrm{~mL}, 1.8 \mathrm{mmol})$ were used. The procedure was the same as that for $\alpha$-CD.

\section{Characterization}

Differential scanning calorimetry (DSC) of the material was performed under a continuous nitrogen flow $(60 \mathrm{~mL} / \mathrm{min})$ with a TA Instrument DSC 2910. Each sample was first heated to $200{ }^{\circ} \mathrm{C}$ and maintained isothermally for $5 \mathrm{~min}$ and then was quickly quenched to $-110{ }^{\circ} \mathrm{C}$. Data were gathered during the second heating cycle at a scanning rate of $20{ }^{\circ} \mathrm{C} / \mathrm{min}$ over a temperature range of -110 to $200{ }^{\circ} \mathrm{C}$. The glasstransition temperature was taken as the midpoint of the heat capacity transition between the upper and lower points of the deviation from the extrapolated glass and liquid lines.

Thermogravimetric analysis (TGA) was performed under nitrogen or air with a TA Instrument TGA 2050 thermogravimetric analyzer at a heating rate of $20{ }^{\circ} \mathrm{C} / \mathrm{min}$ from room temperature to $800{ }^{\circ} \mathrm{C}$. The nitrogen or air flow rate was $60 \mathrm{~mL} / \mathrm{min}$.

FTIR spectra were obtained with a Nicolet Avatar 320 FTIR spectrometer; 32 scans were collected with a spectral resolution of $1 \mathrm{~cm}^{-1}$. The conventional $\mathrm{KBr}$ disk method was employed. A THF solution containing the sample was cast onto a $\mathrm{KBr}$ disk and dried under conditions similar to those used in the bulk preparation. The holder was placed in the sample chamber, and the spectrum was recorded under a purge of $\mathrm{N}_{2}$ to maintain the film's dryness. The weight-average molecular weight $\left(M_{\mathrm{w}}\right), M_{\mathrm{n}}$, and PDI $\left(M_{\mathrm{w}} /\right.$ $M_{\mathrm{n}}$ ) were measured with a Waters 410 gel permeation chromatography (GPC) system equipped with refractive-index and ultraviolet detectors and a Styragel column $(300 \times 7.8 \mathrm{~mm})$. The system was calibrated with polystyrene standards, the eluent was THF, and the flow rate was $1.0 \mathrm{~mL} / \mathrm{min}$.
${ }^{1} \mathrm{H}$ NMR spectroscopy analyses were performed with a Varian Unity Inova-500 NMR spectrometer at $500 \mathrm{MHz}$. All spectra were recorded with $\mathrm{CDCl}_{3}$ as the solvent and tetramethylsilane as the external standard. The ${ }^{13} \mathrm{C}$ cross-polarization/magic-angle-spinning (CP-MAS) NMR spectra were recorded at room temperature with a Bruker DSX-400WB NMR spectrometer at a sample spinning rate of $8.0 \mathrm{kHz}$. The spectra were acquired with a $3.9-\mu$ s proton $90^{\circ}$ pulse, a 1-ms contact time, and a 3-s repetition time.

X-ray diffraction (XRD) measurements were performed with the BL01B wiggler beam line of the National Synchrotron Radiation Research Center of Taiwan. A triangular, bent $\mathrm{Si}(111)$ single crystal was used to obtain a monochromated beam with a wavelength of $\lambda=0.775 \AA$; XRD patterns were collected with Fuji BAS III imaging plates (IPs; area $=20 \times 40 \mathrm{~cm}^{2}$ ) curved with a radius equivalent to the sample-todetector distance $(335 \mathrm{~mm})$. With the $100-\mu \mathrm{m}$ pixel resolution of the IPs and a typical exposure time of $10 \mathrm{~min}$, the XRD data were measured in the $2 \theta$ range of $2-40^{\circ}$. The two-dimensional powder diffraction patterns observed for the samples (typical diameter $=10 \mathrm{~mm}$, thickness $=1 \mathrm{~mm}$ ) were circularly averaged to onedimensional diffraction profiles of $I(2 \theta)$, with the $2 \theta$ values calibrated with a standard Si powder.

Scanning electron microscopy (SEM) images were taken with a Toshiba S4700I field emission microscope working at a voltage of $5 \mathrm{kV}$ and a beam current of $1 \times 10^{-10} \mathrm{~A}$. The sample was coated with a thin Pt layer to increase the contrast and quality of the images.

\section{RESULTS AND DISCUSSION}

\section{Synthesis of $\mathrm{mPPCL}$}

POSS reagents containing one or two points of functionalization are desirable for the design of linear, polymeric POSS systems. To synthesize the organic-inorganic hybrid polymer through the ring-opening polymerization of lactone, we attached a hydroxyl group to the cubic silsesquioxane. Corner capping of the incompletely condensed POSS trisilanols $\left[\mathrm{R}_{7} \mathrm{~T}_{7}(\mathrm{OH})_{3}\right]$ is an efficient method for preparing such a reagent. ${ }^{34}$ It can be carried out with a variety of trichlorosilane coupling agents to produce a fully condensed $\mathrm{T}_{8}$-POSS silicon-oxygen framework. The prepared $\mathrm{T}_{8}-$ POSS reagent possesses only 


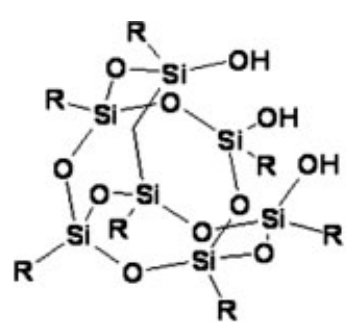

$\mathbf{R}=\mathbf{i}-$ butyl

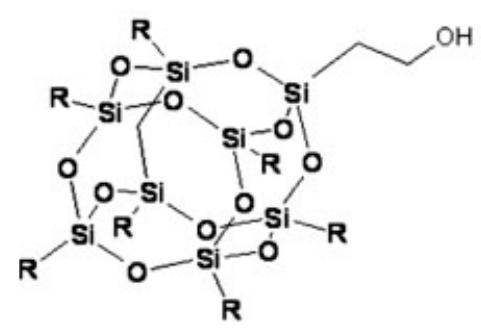

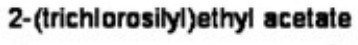

$3 \mathrm{NEt}_{3}, \mathrm{THF}$
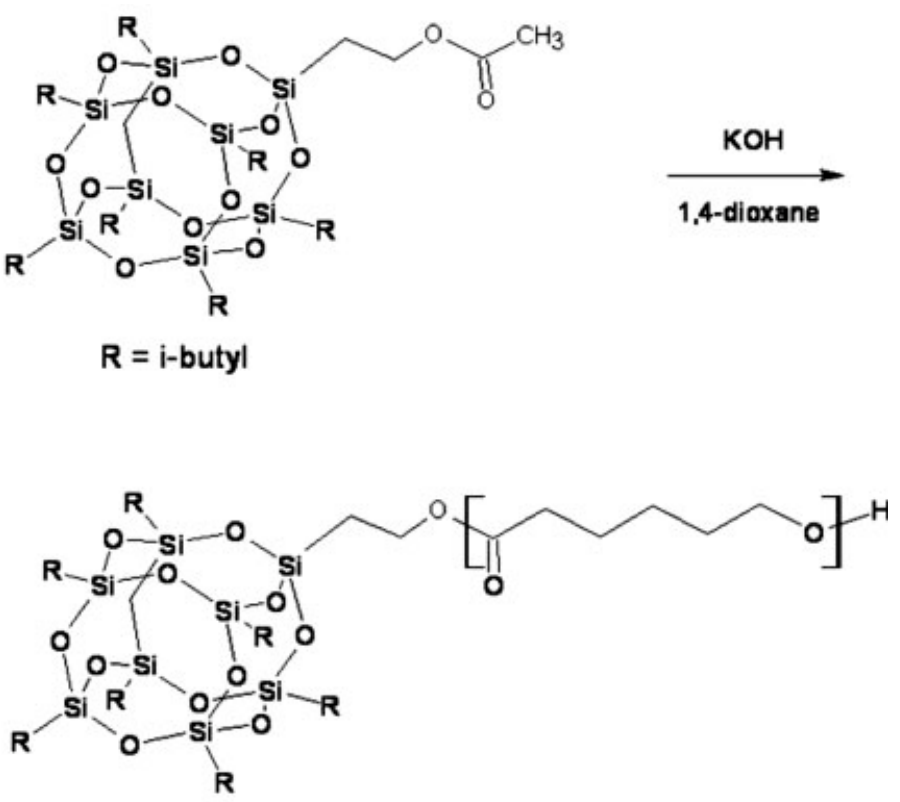

Scheme 1. Synthesis of mPPCL.

one functionalized corner with a polymerizable group. Scheme 1 summarizes the general approach used to synthesize mPPCL. The precursor of the monofunctionalized initiator was synthesized by the reaction of 2-(trichlorosilyl)ethyl acetate with the silanol groups of trisilanolisobutyl-POSS. Figures 1 and 2 show the ${ }^{1} \mathrm{H}$ NMR and FTIR spectra of various related products. Both the ${ }^{1} \mathrm{H}$ NMR and FTIR spectra confirmed that the desired product was successfully produced and possessed only one arm with ester functionality from its cubic silsesquioxane [Figs. 1(b) and 2(b)]. After hydrolyzation of the carbonyl group of the precursor, monohydroxy-functionalized POSS was obtained, as evidenced by ${ }^{1} \mathrm{H}$ NMR and FTIR spectroscopy [Figs. 1(c) and 2(c)].

The polymerization conducted in this work was performed with the hydroxyethylisobutylPOSS derivative, which contained only one primary alcohol functionality as the initiation center for the living ring-opening polymerization of cyclic $\varepsilon$-CL. The FTIR spectra show that the signal of the hydroxyl group (3200-3500 $\mathrm{cm}^{-1}$ ) of the initiator hydroxyethylisobutyl-POSS disappeared, a signal for the carbonyl group of the PCL appeared $\left(1733 \mathrm{~cm}^{-1}\right)$, and the characteristic POSS signals were preserved [Fig. 2(d)]. The molecular weight of mPPCL was about $6100($ PDI $=1.2)$.

Journal of Polymer Science: Part A: Polymer Chemistry DOI 10.1002/pola

\section{Formation of the ICs}

The ICs that form between linear PCL and CDs have been studied on a number of occasions. ${ }^{35}$ Similar to its uncapped PCL analogue, mPPCL is able to form ICs in high yields with both $\alpha$-CD and $\gamma-\mathrm{CD}$ and in a moderate yield with $\beta$-CD. ${ }^{36}$ This is due to the close correlation of the crosssectional area of the all-trans conformation of the PCL polymer chain and the cavity sizes of the $\alpha$ - and $\gamma$-CDs. ${ }^{37}$ It is well known that the stoichiometry of the ICs is $1: 1$ ( $\varepsilon$-CL unit to CD) for the $\alpha$-CD/PCL ICs and 2:1 for the $\gamma$-CD/PCL ICs; that is, the $\alpha$-CD/PCL IC contains a single chain in each $\alpha$-CD channel, whereas the $\gamma$-CD/ PCL IC contains two adjacent PCL chains in the same cavity of $\gamma$-CD. ${ }^{8}$ When an aqueous solution of $\alpha$-CD or $\gamma$-CD is added to mPPCL soaked with acetone and sonicated for $10 \mathrm{~min}$, complexes are formed as crystalline precipitates, indicating the formation of a crystalline IC between the polymer and the CD.

The formation of CD/mPPCL ICs has been further verified by XRD studies. XRD patterns of the $\alpha-\mathrm{CD}, \alpha-\mathrm{CD} / \mathrm{mPPCL}$ IC, $\gamma-\mathrm{CD}$, and $\gamma-\mathrm{CD} /$ mPPCL IC, presented in Figure 3, clearly reveal that crystalline ICs are indeed formed between CDs and mPPCL instead of a mixture of these components. The diffraction pattern of pure 


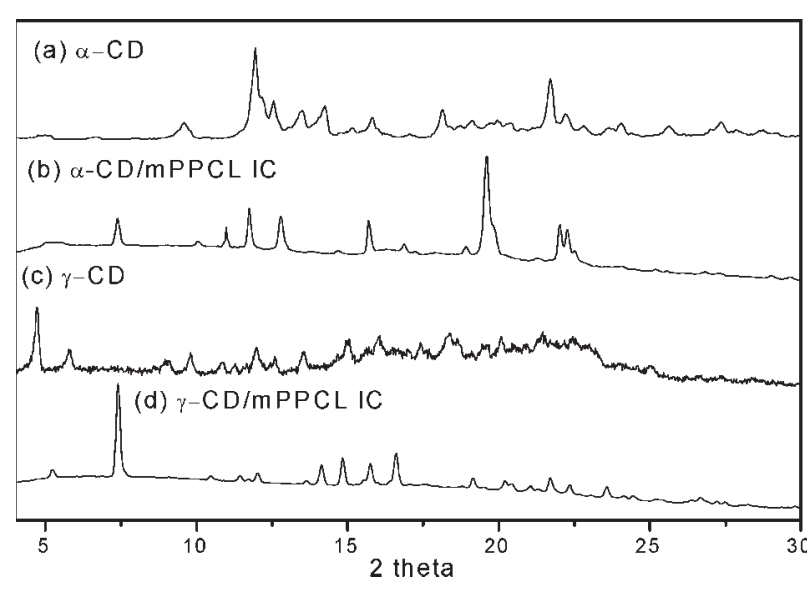

Figure 3. XRD patterns of (a) $\alpha-\mathrm{CD}$, (b) $\alpha-\mathrm{CD} /$ mPPCL IC, (c) $\gamma$-CD, and (d) $\gamma$-CD/mPPCL IC.

mPPCL possesses very strong crystalline diffraction peaks at 21.3 and $23.6^{\circ}$ from the PCL segments (not shown here). The patterns of these two synthesized ICs [Fig. 3(b,d)] are substantially different from that of pure mPPCL. Obviously, these ICs are not merely a simple superposition of $\mathrm{mPPCL}$ and its respective CD components. Normally, a columnar structure is assigned to the ICs of linear chain polymers with CDs. For the $\alpha-\mathrm{CD} / \mathrm{mPPCL}$ IC, no peak corresponding to the PCL crystalline phase has been detected in the diffractogram [Fig. 3(b)], whereas a prominent peak can be observed at $2 \theta=20^{\circ}$, which is typical for an $\alpha-\mathrm{CD} /$ polymer IC. ${ }^{38,39}$ The appearance of the characteristic peak at $2 \theta=20^{\circ}$ indicates the formation of the complex between $\alpha$-CD and mPPCL. A columnar structure is formed with a radius of about $5 \AA$, rather than a cage structure. ${ }^{8}$ Meanwhile, in the $\gamma$-CD IC system, the most intense diffraction peak in the diffractogram of the $\gamma$-CD columnar structure appears around $7.5^{\circ}$, representing the (200) plane of the hydrated crystals. ${ }^{40}$ This is a fingerprint in the X-ray diffractograms for all $\gamma$-CD IC crystals obtained with long guest molecules. The X-ray results obtained for the $\gamma-\mathrm{CD} /$ mPPCL IC are shown in Figure 3(d). A sharp peak at $2 \theta=7.5^{\circ}$ in the pattern is strong evidence that $\gamma$-CD hosts mPPCL in a channelstructure IC, suggesting that $\gamma$-CD molecules are stacked along an mPPCL axis to form a cylinder instead of a cage-type packing arrangement. The main parts of the two IC patterns discussed previously are essentially comparable to the diagrams of both $\alpha$-CD/linear-PCL and
$\gamma$-CD/linear-PCL ICs, ${ }^{41}$ implying that both polypseudorotaxanes reported here are mainly composed of crystalline IC domains. They are isomorphous, with the channel-type structure formed by their corresponding CD/linear-PCL ICs. These results confirm that both $\mathrm{CD} / \mathrm{mPPCL}$ ICs in this work possess a columnar structure and that the incorporation of bulky POSS moieties causes no apparent change in the unit cell dimensions and hence the crystal structure.

Solid-state ${ }^{13} \mathrm{C}$ CP-MAS NMR analyses provide additional evidence concerning the structures of these two synthesized ICs. Figure 4 displays the solid-state ${ }^{13} \mathrm{C}$ NMR CP-MAS NMR spectra of the $\alpha-\mathrm{CD}, \alpha-\mathrm{CD} / \mathrm{mPPCL}$ IC, $\gamma-\mathrm{CD}$, and $\gamma-\mathrm{CD} / \mathrm{mPPCL}$ IC. The spectrum of pure $\alpha$-CD presents resolved resonances because of the asymmetric glucopyranosyl conformation. The spectrum of the $\alpha-\mathrm{CD} /$ mPPCL IC displays sharp, unresolved singlet resonances, indicating that $\alpha$-CD takes the symmetric cyclic conformation so that each glucose unit exists in a similar environment in the IC. ${ }^{42}$ The spectra of the $\gamma$-CD and $\gamma$-CD/mPPCL IC, presented on the right side, indicate that the IC adopts a symmetrical cyclic conformation in the complex. $\gamma$-CD assumes a less symmetrical conformation in the crystal when it does not include a guest in its cavity. ${ }^{40,41}$ Similar observations from solid-state ${ }^{13} \mathrm{C}$ CP-MAS NMR spectra of ICs prepared from a number of other polymers and CDs previously reported also support the formation of ICs between CDs and polymers. ${ }^{43,44}$ The ${ }^{13} \mathrm{C}$ CP-MAS NMR spectra of the complexed

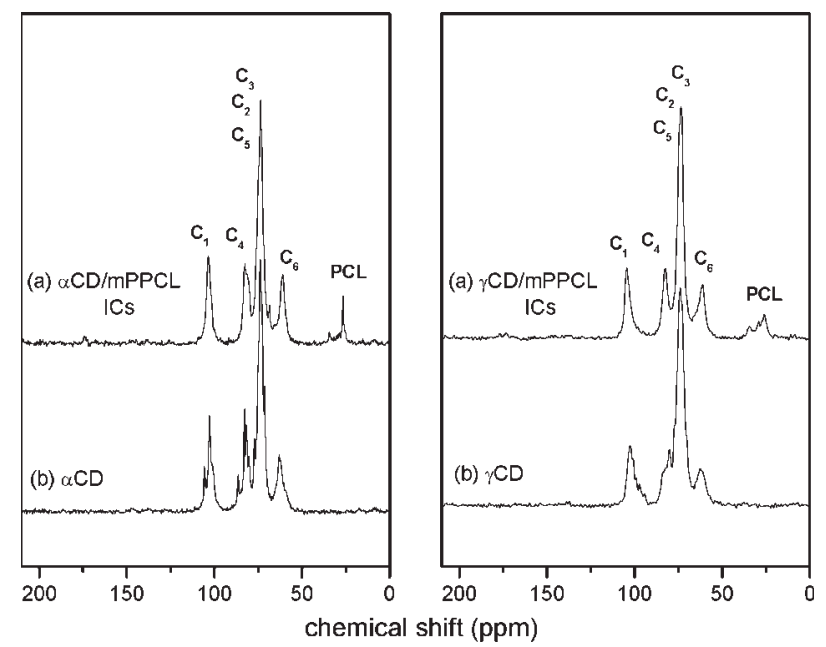

Figure 4. ${ }^{13} \mathrm{C}$ CP-MAS NMR spectra of (a) $\alpha$-CD and (b) $\alpha-\mathrm{CD} / \mathrm{mPPCL}$ IC (left) and (a) $\gamma-\mathrm{CD}$ and (b) $\gamma$-CD/mPPCL IC (right).

Journal of Polymer Science: Part A: Polymer Chemistry 


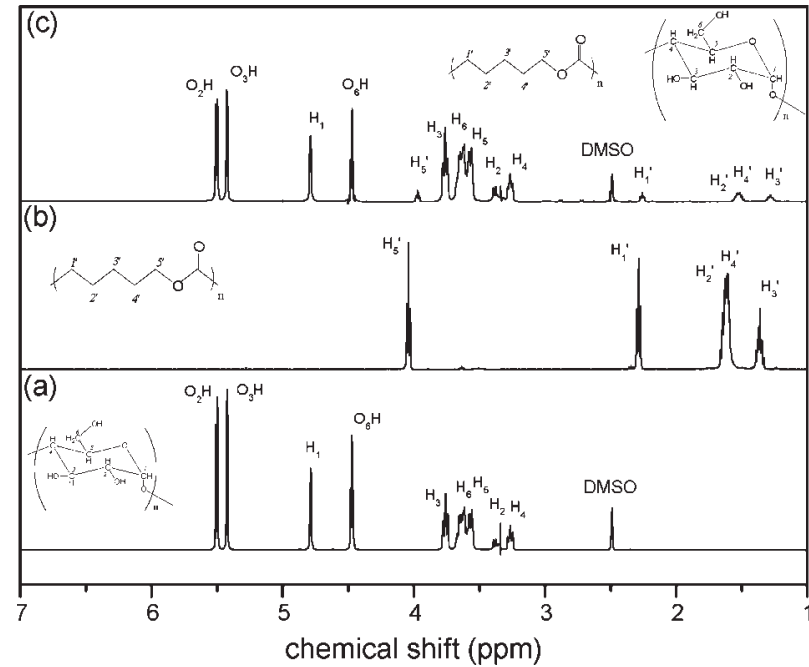

Figure 5. ${ }^{1} \mathrm{H}$ NMR spectra of (a) $\alpha-\mathrm{CD}$, (b) mPPCL, and (c) $\alpha-\mathrm{CD} / \mathrm{mPPCL}$ IC.

and uncomplexed CDs are also consistent with the results of our XRD studies. Therefore, we believe that the PCL chains of mPPCL are included within the cavities of the CDs in a columnar form.

The compositions of the ICs have been characterized with ${ }^{1} \mathrm{H}$ NMR spectroscopy. Figure 5 displays ${ }^{1} \mathrm{H}$ NMR spectra of pure $\alpha$-CD, mPPCL, and the IC that formed between $\alpha-C D$ and mPPCL. The IC [Fig. 5(c)] displays peaks corresponding to both $\alpha$-CD and mPPCL [Fig. 5(a,b)]. By comparing the integral of a peak for $\alpha-C D$ (1H) with that of the PCL methylene group, we find that approximately 1.2 monomer units of $\varepsilon$-CL are bound to one $\alpha$-CD molecule. Because an $\varepsilon-C L / \alpha-C D$ ratio of $1: 1$ is expected for the $\alpha-\mathrm{CD} /$ /inear-PCL IC according to previous literature ${ }^{19}$ these long polymer chains have been included inside the channels provided by the orderly stacked CD molecules. On the other hand, the observed average number of $\varepsilon-\mathrm{CL}$ repeat units bound to one $\gamma$-CD molecule in the $\gamma-\mathrm{CD} / \mathrm{mPPCL}$ IC is 2.5 , in contrast to the stoichiometry for the $\gamma$-CD/linear-PCL IC $(\varepsilon-\mathrm{CL} / \mathrm{CD}$ $=2: 1) .{ }^{19}$ Our results imply that the obtained IC is not in perfect stoichiometry, suggesting that portions of these $\varepsilon$-CL units are not included within the CD channels. The POSS chain end of mPPCL creates steric hindrance on the incoming CDs and leads to stoichiometries higher than 1:1 and 2:1 for $\alpha$ - and $\gamma$-CD ICs, respectively. Steric hindrance is especially pronounced in the $\gamma-\mathrm{CD} /$ mPPCL IC, which usually contains double PCL chains in the same $\gamma$-CD channel.

Journal of Polymer Science: Part A: Polymer Chemistry DOI 10.1002/pola
The thermal properties of the ICs have been characterized with DSC measurements and compared with their respective constituent polymers (data not shown here). The pure mPPCL displays a strong melting peak around $58{ }^{\circ} \mathrm{C}$, and the crystallinity of the mPPCL sample $\left(\Delta \chi_{\mathrm{c}, \mathrm{PCL}}\right)^{45}$ has been calculated to be $54.6 \%$, lower than that of uncapped PCL $(67 \%)^{46}$ because of the stiffening and binding effects exerted by the introduction of the bulky POSS cap. On the other hand, we have not observed any fusion peak from the $\alpha-\mathrm{CD} / \mathrm{mPPCL}$ IC in the heating run, and this indicates that the original crystalline polymer phase of mPPCL is missing in the $\alpha-\mathrm{CD} / \mathrm{mPPCL}$ IC. ${ }^{47}$ On the contrary, a weak melting point has been observed around $55{ }^{\circ} \mathrm{C}$ from the $\gamma$-CD/ mPPCL IC with a crystallinity of about $5 \%$. This result demonstrates that the crystallization of PCL chains has been significantly inhibited or retarded by the formation of ICs. Further verification by ${ }^{1} \mathrm{H}$ NMR reveals that a fraction of the PCL chains of mPPCL in the $\gamma-\mathrm{CD} / \mathrm{mPPCL}$ IC are not included within the columnar structure of the IC and thus are able to form minor crystalline domains. However, the crystalline regions formed by the uncomplexed mPPCL chains represent just a small fraction of the entire sample; therefore, no diffraction peak corresponding to free $\mathrm{mPPCL}$ is expected in the corresponding XRD pattern.

Figure 6 shows FTIR spectra of mPPCL, the $\alpha-\mathrm{CD} / \mathrm{mPPCL}$ IC, and the $\gamma-\mathrm{CD} / \mathrm{mPPCL}$ IC. The

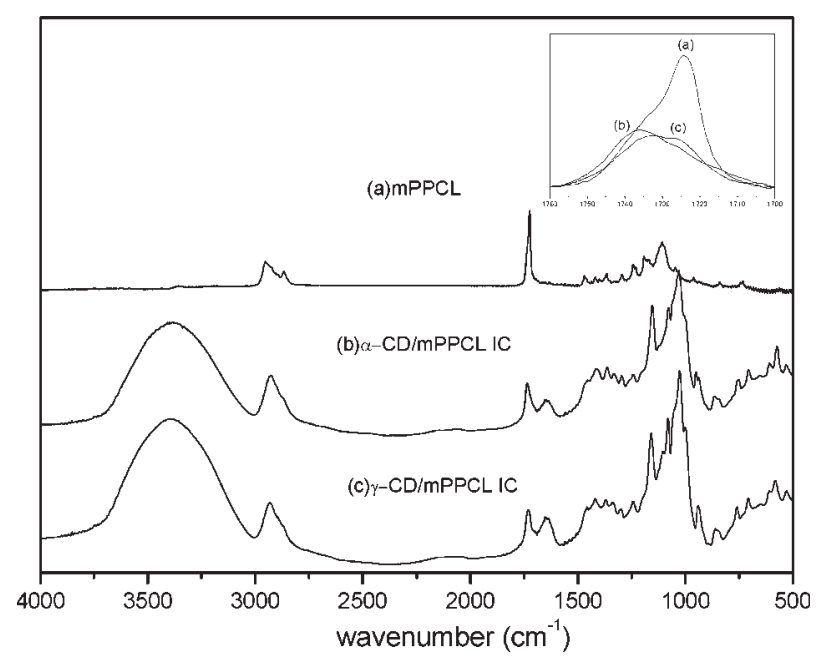

Figure 6. FTIR spectra of (a) mPPCL, (b) $\alpha-\mathrm{CD} /$ mPPCL IC, and (c) $\gamma-C D / m P P C L$ IC. The inset presents an expansion of the carbonyl absorption region of (a) mPPCL, (b) $\alpha-\mathrm{CD} / \mathrm{mPPCL}$ IC, and (c) $\gamma$-CD/mPPCL IC. 
Table 1. Summary of the Thermal Characteristics of the CDs, Synthesized mPPCL, and Their ICs

\begin{tabular}{|c|c|c|c|c|c|c|c|}
\hline & \multicolumn{3}{|c|}{$T_{\mathrm{d}, \text { free }}\left({ }^{\circ} \mathrm{C}\right)^{\mathrm{a}}$} & \multicolumn{2}{|c|}{$T_{\text {d,complexes }}\left({ }^{\circ} \mathrm{C}\right)^{\mathrm{b}}$} & \multicolumn{2}{|c|}{$T_{\text {d,complexes }}\left({ }^{\circ} \mathrm{C}\right)^{\mathrm{b}}$} \\
\hline & $\alpha-\mathrm{CD}$ & $\gamma-\mathrm{CD}$ & mPPCL & $\alpha-\mathrm{CD}$ & mPPCL & $\gamma-\mathrm{CD}$ & $\mathrm{mPPCL}$ \\
\hline $\mathrm{CD} / \mathrm{mPPCL} \mathrm{IC}$ & 310 & 314 & 360 & 317 & 386 & 316 & 378 \\
\hline
\end{tabular}

broad hydroxyl band at $3360 \mathrm{~cm}^{-1}$ of pure $\alpha-\mathrm{CD}$ shifts to a high frequency at $3390 \mathrm{~cm}^{-1}$ after a supramolecular structure is formed. This shift most likely results from the hydrogen-bonding interaction between hydroxyl groups of $\alpha$-CDs and the included PCL backbone. The most distinct band in the spectra of the ICs is at $1733 \mathrm{~cm}^{-1}$, the stretching of the carbonyl groups of PCL. The carbonyl absorption band of pure mPPCL is well resolved into two bands corresponding to a crystalline peak at $1726 \mathrm{~cm}^{-1}$ and a noncrystalline shoulder absorption at $1736 \mathrm{~cm}^{-1}{ }^{47}$ In the complexed form, the PCL chain is situated orderly within the IC channel and thus is unable to aggregate to form PCL crystals; that is, the peak at $1726 \mathrm{~cm}^{-1}$ is significantly depressed in the spectra of ICs. ${ }^{48,49}$ The expansion of the carbonyl region of FTIR spectra of $\mathrm{CD} / \mathrm{mPPCL}$ ICs provides useful information concerning the shifting of the phase structure. The inset of Figure 6 presents an expansion of the carbonyl region of the spectra of pure mPPCL, the $\alpha$-CD/mPPCL IC, and the $\gamma$-CD/ mPPCL IC. A comparison of the spectrum of pure $\mathrm{mPPCL}$ and the spectrum of the $\alpha-\mathrm{CD} /$ mPPCL IC (inset) shows that the carbonyl absorption of the crystalline PCL region (1726 $\mathrm{cm}^{-1}$ ) has nearly disappeared, and only the carbonyl absorption corresponding to the noncrystalline PCL $\left(1736 \mathrm{~cm}^{-1}\right)$ can be detected. This result agrees well with those obtained from DSC and ${ }^{1} \mathrm{H}$ NMR: no crystalline PCL phase exists in the $\alpha$-CD ICs, and all PCL blocks are involved in the inclusion process to form a columnar structure. However, in the spectra of the $\gamma-\mathrm{CD} / \mathrm{mPPCL}$ IC, the noncrystalline absorption is increased, whereas the absorption of the crystalline PCL region is substantially reduced, but not totally gone.

These ICs are thermally stable. ${ }^{50}$ TGA measurements recorded up to $600{ }^{\circ} \mathrm{C}$ for the pure
$\mathrm{CDs}, \mathrm{mPPCL}$, and their respective ICs are summarized in Table 1. These ICs show two distinct decomposition transitions, corresponding to the decompositions of CDs and mPPCL separately. The onset decomposition temperatures of $\alpha$-CD and MPPCL in the IC can be observed at 317 and $386{ }^{\circ} \mathrm{C}$, which are higher than those in the free state $\left(310\right.$ and $\left.360{ }^{\circ} \mathrm{C}\right)$. Similar results have also been observed for the $\gamma$-CD/mPPCL IC system. mPPCL in all the ICs has a decomposition temperature higher than that of the pure form (a shift from about 360 to $380{ }^{\circ} \mathrm{C}$ ); this indicates that complexation with the CDs enhances the thermal stability of mPPCLs. ${ }^{51}$ The thermal stabilities of the CDs in their IC states are also higher than those of the pure CDs, and this implies that the presence of guests included within the CD channels can improve the thermal stability of the original CDs.

Although the $\gamma-\mathrm{CD} / \mathrm{mPPCL}$ IC still contains some PCL crystals, as revealed by DSC and FTIR, it is reasonable to assume that the fraction of the free PCL phase is relatively minor, and the size of the PCL crystalline domains is quite small. The crystalline structures of these two ICs can basically be determined as the channel type according to XRD and ${ }^{13} \mathrm{C}$ CP-MAS NMR spectroscopy. The $\alpha-\mathrm{CD} / \mathrm{mPPCL}$ IC contains a single PCL chain in each $\alpha$-CD channel, whereas the $\gamma$-CD/mPPCL IC contains two PCL chains; that is, they possess architectures similar to those found in the frameworks of the corresponding CD/linear-PCL ICs.

Normally, the crystals of ICs of $\alpha$ - and $\gamma$-CDs with polymeric chains show well-edged, parallelepiped shapes. ${ }^{52}$ The morphologies of $\alpha$-CD/PEO complexes have been reported. ${ }^{53-55}$ Hexagonal fibers ${ }^{53}$ and flakes ${ }^{54}$ have been formed separately in the complexation of linear $\mathrm{PEO}$ and very densely PEO-grafted polymer brushes. Moreover, a multiarm, hyperbranched polyether complexed 

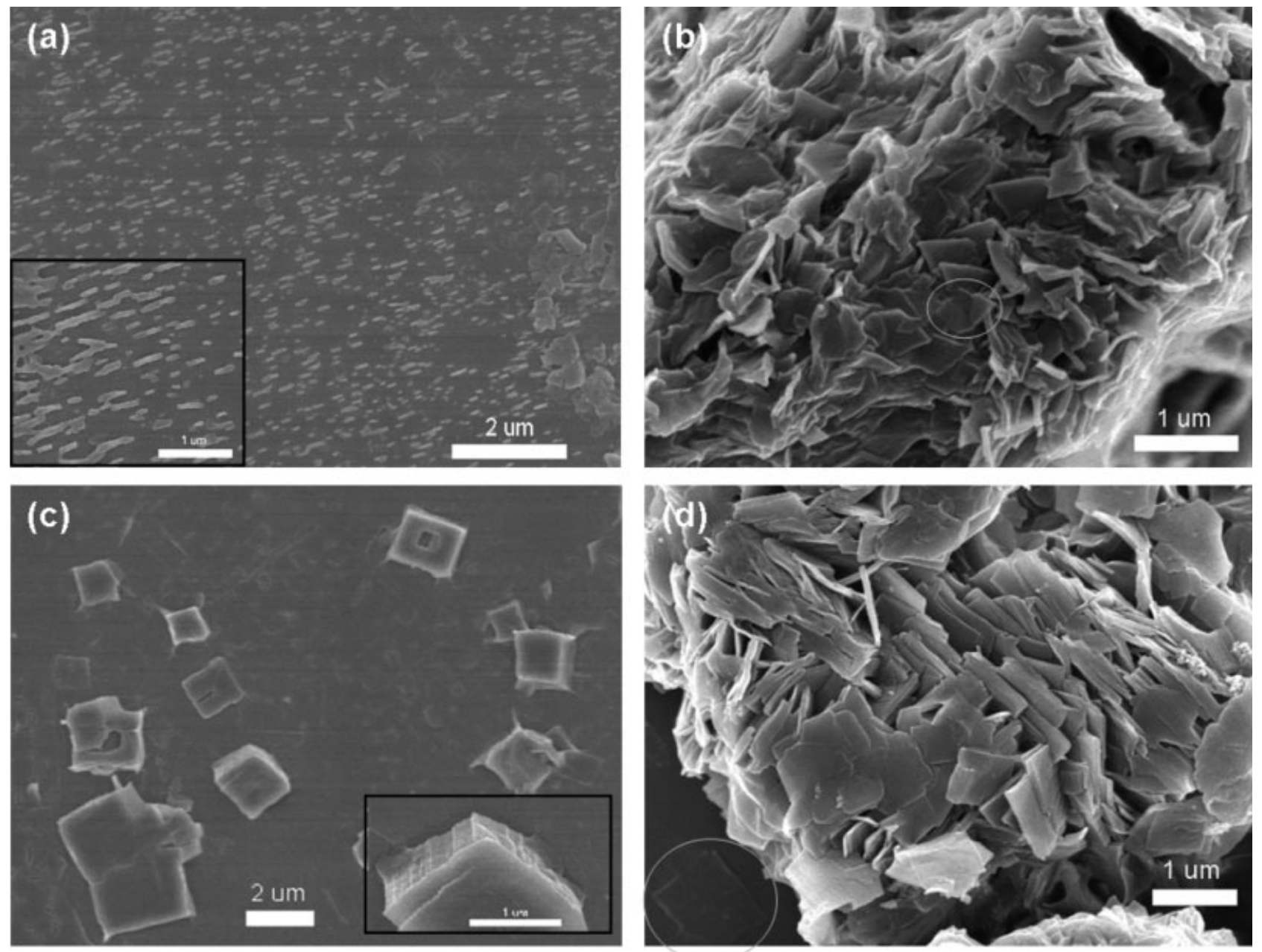

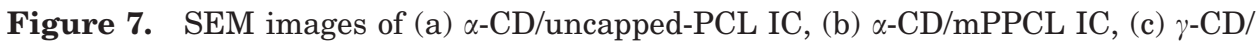
uncapped-PCL IC, and (d) $\gamma$-CD/mPPCL IC.

with $\alpha$-CDs shows a novel lamellar morphology with a juxtaposed manner. ${ }^{55}$ In this work, we have investigated the effect of the bulky POSS moiety on the resultant morphologies of these $\mathrm{CD} / \mathrm{mPPCL}$ IC systems. $\alpha$-CD/linear-PCL and $\gamma$-CD/linear-PCL ICs $\left(M_{\mathrm{n}} \fallingdotseq 6000\right.$, PDI $\left.=1.2\right)$ are used for comparison. In all the aforementioned cases, the crystalline-inclusion precipitates were again dispersed in THF. The suspensions of these precipitates that formed were dropped onto mica, and the morphologies of these crystals were characterized with SEM directly. Figure 7 depicts the morphological change from the $\mathrm{CD} /$ linear-PCL ICs to the CD/mPPCL ICs. Although all the ICs display a platelet, lamellar structure resulting from the aggregation of columnar molecules, the morphologies of the $\mathrm{CD} / \mathrm{mPPCL}$ ICs show more regularity in large aggregates.
Figure 7(a) shows the SEM images of the $\alpha-C D /$ linear-PCL IC. The IC is a platelet structure standing perpendicular to the surface with good alignment [see the insert of Fig. 7(a)]. The thickness of each platelet is about $60 \mathrm{~nm}$, and the length of the edge ranges from 150 to $800 \mathrm{~nm}$. On the contrary, the $\alpha-C D / m P P C L$ IC selfassembles into a large stack of monoclinically shaped flakes with close packing [Fig. 7(b)]. According to the literature, ${ }^{52}$ we speculate that the formation of the monoclinic flakes is probably caused by the crystallized crystal with hexagonal, lateral packing of the molecular columns, with their axes tilted at the basal plane. Unlike the variation of the platelet size structures of the $\alpha-C D /$ linear-PCL IC, these parallelogram flakes are almost equal in size, with a width of about $400 \mathrm{~nm}$. On the other 
(a)

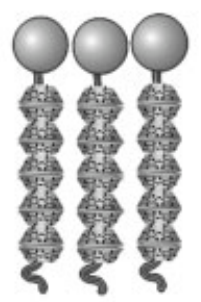

(b)

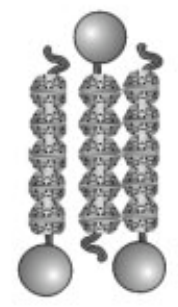

(c)

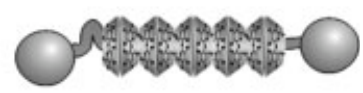

(d)

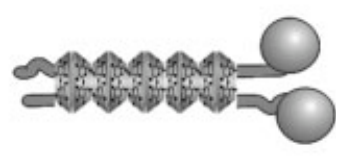

\section{- POSS

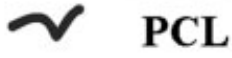 and CDs}

Scheme 2. Possible structures of the supramolecular-assembly single layers of the $\alpha-\mathrm{CD} / \mathrm{mPPCL}$ ICs and $\gamma-\mathrm{CD} / \mathrm{mPPCL}$ ICs.

hand, the $\gamma$-CD/linear-PCL IC is crystallized in a tetragonal form. As shown in Figure 7(c), most of the squares, composed of piles of tetragonal flakes, retain individually to each other; the width of the squares is $1-2.2 \mu \mathrm{m}$. The morphology of the $\gamma-\mathrm{CD} / \mathrm{mPPCL}$ IC is revealed in Figure $7(\mathrm{~d})$. Although the aggregates of the $\gamma-\mathrm{CD} /$ mPPCL IC also consist of plenty of tetragonal squares, they show a more uniform tilt and close packing. The size of each slice is almost normalized with a width of $1 \mu \mathrm{m}$. Again, similar to the aggregates of the $\alpha$-CD/mPPCL IC, those in the $\gamma$-CD/mPPCL IC exhibit higher regularity than the CD/linear-PCL ICs.

According to the previous discussion, it can be concluded that the introduction of POSS moieties into $\mathrm{CD} / \mathrm{mPPCL}$ IC systems induces morphological regularity. This result is probably caused by the presence of the POSS moiety, which itself tends to be aggregated into a closepacking, hexagonal, crystalline structure ${ }^{56}$ and consequently provides a driving force during the self-assembly. Thus, with a POSS moiety incorporated into the IC channel, the CD/mPPCL ICs can be self-assembled in THF because THF is a good solvent for POSS and PCL but a poor solvent for ICs formed between CDs and mPPCL. The self-assemblies of the $\alpha-\mathrm{CD} / \mathrm{mPPCL}$ and $\gamma$-CD/mPPCL ICs in THF lead to the formation of more regular, lamellar, crystalline precipitates. Scheme 2 illustrates possible structures of the $\alpha-\mathrm{CD} / \mathrm{mPPCL}$ ICs (structures a and $b$ ) and $\gamma-\mathrm{CD} / \mathrm{mPPCL}$ ICs (structures c and d). Because $\gamma$-CD/mPPCL ICs tend to form tetragonal crystals, structure d seems less favorable because of the steric hindrance of these bulky POSS groups gathering on one side.

\section{CONCLUSIONS}

ICs of mPPCL with CDs have been developed. Similarly to the findings reported previously for linear-PCL analogues, star PCL forms ICs with $\alpha$ - and $\gamma$-CDs but not with $\beta$-CD. These CD ICs have been characterized with various analytical methods. XRD and solid-state ${ }^{13} \mathrm{C}$ CP-MAS NMR spectroscopy analyses have indicated that these mPPCL chains are confined within these CD channels as columnar, crystalline structures. The PCL/CD ratios for all the ICs investigated here with $\alpha$ - and $\gamma$-CDs are greater stoichiometries because of the steric hindrance of the bulky POSS moiety, and some of the $\varepsilon$-CL units are left free from developing the ICs and form minor crystalline domains, as evidenced by FTIR spectroscopy and DSC measurements. The morphologies of the ICs have been examined with SEM. According to our results, $\alpha$-CD/mPPCL and $\gamma$ $\mathrm{CD} / \mathrm{mPPCL}$ ICs in THF lead to the formation of more regular, lamellar crystals than $\mathrm{CD} /$ /inearPCL ICs.

This research was supported financially by the National Science Council of Taiwan, Republic of China, under contract NSC-95-2216-E-009-001 and by the Ministry of Education through the program "Aim for the Top University".

\section{REFERENCES AND NOTES}

1. Harada, A.; Li, J.; Kamachi, M. Nature 1993, 364, 516.

2. Lo Nostro, P.; Lopes, J. R.; Ninham, B. W.; Banglion, P. J Phys Chem B 2002, 106, 2166.

3. Okada, M.; Takashima, Y.; Harada, A. Macromolecules 2004, 37, 7075.

Journal of Polymer Science: Part A: Polymer Chemistry DOI 10.1002/pola 
4. Liu, Y.; Zhao, Y. L.; Zhang, H. Y.; Li, X. Y.; Liang, P.; Zhang, X. Z.; Xu, J. J. Macromolecules 2004, 37, 6362 .

5. Jiao, H.; Goh, S. H.; Valiyaveettil, S. Macromolecules 2002, 35, 1399.

6. Wenz, G. Angew Chem Int Ed Engl 1994, 33, 803.

7. Rusa, C. C.; Bullions, T. A.; Fox, J.; Porbeni, F. E.; Wang, X.; Tonneli, A. E. Langmuir 2002, 18, 10016.

8. Okumura, H.; Kawaguchi, Y.; Harada, A. Macromolecules 2003, 36, 6422 .

9. Topchieva, I. N.; Tonelli, A. E.; Panova, I. G.; Matuchina, E. V.; Kalashnikov, F. A.; Gerasimov, V. I.; Rusa, C. C.; Rusa, M.; Hunt, M. A. Langmuir 2004, 20, 9036.

10. Li, J.; Ni, X.; Leong, K. Angew Chem Int Ed 2003, 42, 69.

11. Jiao, H.; Goh, S. H.; Valiyaveettil, S. Macromolecules 2003, 36, 4241.

12. Zhu, X.; Chen, L.; Yan, D.; Chen, Q.; Yao, Y.; Xiao, Y.; Hou, J.; Li, J. Langmuir 2004, 20, 484.

13. Hwang, M. J.; Bae, H. S.; Kim, S. J.; Jeong, B. Macromolecules 2003, 37, 8820.

14. Fujita, H.; Ooya, T.; Yui, N. Macromolecules 1999, 32, 2534

15. Ceccato, M.; Lo Nostro, P.; Baglioni, P. Langmuir 1997, 13, 2436.

16. Harada, A.; Li, J.; Kamachi, M. Nature (London) 1994, 370, 126.

17. Harada, A.; Kamachi, M. Macromolecules 1990, 23, 2821.

18. Harada, A. Coord Chem Rev 1996, 148, 115.

19. Huang, L.; Allen, E.; Tonelli, A. E. Polymer 1998, $39,4857$.

20. Nasanthan, N.; Tonelli, A. E.; Nojima, S. Macromolecules 1994, 27, 7220.

21. Huang, L.; Allen, E.; Tonelli, A. E. Polymer 1999, 40, 3211.

22. Constable, G. S.; Lesser, A. J.; Coughlin, E. B. Macromolecules 2004, 37, 1276.

23. Xu, H.; Kuo, S. W.; Lee, J. L.; Chang, F. C. Macromolecules 2002, 35, 8788.

24. Leu, C. M.; Chang, Y. T.; Wei, K. H. Chem Mater 2003, 15, 3721.

25. Fu, B. X.; Lee, A.; Haddad, T. S. Macromolecules 2004, 37, 5211.

26. Zhang, C.; Laine, R. M. J Am Chem Soc 2000, $122,6979$.

27. Li, G.; Wang, L.; Ni, H.; Pittman, C. U., Jr. J Inorg Organomet Polym 2001, 11, 123.

28. Nouvel, C.; Ydens, I.; Degee, P.; Dubois, P.; Dellacherie, E.; Six, J. L. Macromol Symp 2001, $175,33$.

29. Kawaguchi, Y.; Nishiyama, T.; Okada, M.; Kamachi, M.; Harada, A. Macromolecules 2000, 33,4472 .
30. Lin, H. M.; Wu, S. Y.; Huang, P. Y.; Huang, C. F.; Kuo, S. W.; Chang, F. C. Macromol Rapid Commun 2006, 27, 1550.

31. Cui, L.; Zhu, L. Langmuir 2006, 22, 5982.

32. Cui, L.; Collet, J. P.; Xu, G.; Zhu, L. Chem Mater 2006, 18, 3503.

33. Chan, S. C.; Kuo, S. W.; Chang, F. C. Macromolecules $2005,38,3099$.

34. Lichtenhan, J. D.; Otonari, Y. A.; Carr, M. J. Macromolecules 1995, 28, 8435.

35. Rusa, C. C.; Luca, C.; Tonelli, A. E. Macromolecules 2001, 34, 1318 .

36. Kawaguchi, Y.; Nishiyama, T.; Okada, M.; Kamachi, M.; Harada, A. Macromolecules 2000, 33,4472 .

37. Shin, I. D.; Huang, L.; Tonelli, A. E. Macromol Symp 1999, 138, 21.

38. Huh, K. M.; Ooya, T.; Sasaki, S.; Yui, N. Macromolecules 2001, 34, 2402.

39. Rusa, C. C.; Tonelli, A. E. Macromolecules 2000, 33,5321

40. Takeo, K.; Kuge, T. Agric Biol Chem 1970, 34, 568.

41. Rusa, C. C.; Fox, J.; Tonelli, A. E. Macromolecules 2003, 36, 2742.

42. Gidey, M. J.; Bociek, S. M. J Am Chem Soc 1988, $110,3820$.

43. Harada, A.; Suzuke, S.; Okada, M.; Kamachi, M. Macromolecules 1996, 29, 5611.

44. Hamadan, A. A.; Bugnon, P.; Sandan, C.; Lye, G. P.; Merbach, A. E. J Am Chem Soc 2000, 122, 592.

45. Crezcenzi, V.; Manzini, G.; Cazolari, G.; Borri, C. Eur Polym J 1972, 8, 449.

46. $\Delta \chi_{\mathrm{c}, \mathrm{PCL}}=\Delta H / \Delta H_{0} w$ (weight percentage of PCL), where $\Delta H_{0}$ is the melting enthalpy of pure PCL in the completely crystalline state $(139 \mathrm{~J} / \mathrm{g})$.

47. He, Y.; Inoue, Y. Polym Int 2000, 49, 623.

48. Shuai, X.; Porbeni, F. E.; Wei, M.; Bullions, T.; Tonelli, A. E. Macromolecules 2002, 35, 2401.

49. Shuai, X.; Porbeni, F. E.; Wei, M.; Bullions, T.; Tonelli, A. E. Macromolecules 2002, 35, 3126.

50. Li, J.; Ni, X.; Zhou, Z.; Leong, K. J Am Chem Soc 2003, 125, 1788 .

51. Li, J.; Chenb, B.; Wang, X.; Goh, S. H. Polymer 2004, 45, 1777.

52. Ohmura, M.; Kawahara, Y.; Okude, K.; Hasegawa, Y.; Hayashida, M.; Kurimoto, R.; Kawaguchi, A. Polymer 2004, 45, 6967.

53. Hwang, M. J.; Bae, H. S.; Kim, S. J.; Jeong, B. Macromolecules 2004, 37, 8820 .

54. He, L.; Huang, J.; Chen, Y.; Xu, X.; Liu, L. Macromolecules 2005, 38, 3845.

55. Zhu, X.; Chen, L.; Yan, D.; Chen, O.; Yao, Y.; Xiao, Y.; Hou, J.; Li, J. Langmuir 2004, 20, 484.

56. Zheng, L.; Hong, S.; Cardoen, G.; Burgaz, E.; Gido, S. P.; Coughlin, E. B. Macromolecules 2004 , $37,8606$. 